\title{
Achieving Optimal Correction for Young Myopic Children: A Concept Study
}

\author{
Sara Bøgelund Rasmussen Flemming Møller Trine Møldrup Jakobsen \\ Department of Ophthalmology, Vejle Hospital, University Hospital of Southern Denmark, Vejle, Denmark
}

\section{Keywords}

Myopia · Children · Autorefraction · Subjective manifest refraction

\begin{abstract}
The purpose of this article is to explore alternative ways of achieving optimal correction for myopic children who cannot cooperate to subjective manifest refraction (SR). The study included myopic children aged 9-12 years who underwent non-cycloplegic SR and autorefraction with and without cycloplegia using the Shin-Nippon Nvision-K 5001 autorefractor (AR) as well as non-cycloplegic autorefraction using the Topcon KR-800S AR. There were 21 children (mean age, 10.62 years) included. The spherical equivalent refractive error of SR was not significantly different from that of non-cycloplegic AR measurements, but it was significantly different from that of cycloplegic Shin-Nippon Nvision-K 5001 measurements $(p<0.001)$. Compared with SR, cycloplegic Shin-Nippon Nvision-K 5001 measured a less myopic refractive error (median: $-2.44 \mathrm{D}$ vs. $-2.88 \mathrm{D}, p<0.001$ ). For both ARs, the axis measurements and astigmatic dioptre values between SR and autorefraction were not significantly different. Compared with non-cycloplegic SR, cycloplegic measurements showed a lesser degree of myopic refractive error. There was no significant difference between SR and non-cycloplegic autorefraction. Therefore, the Topcon KR800 S and the Shin-Nippon Nvision-K 5001 ARs may be useful
\end{abstract}

for prescribing glasses in myopic children who cannot cooperate during SR. However, caution should be taken with cylinders $<0.75 \mathrm{D}$ because the agreement in axis between SR and AR measurement is poor. Therefore, in such cases, we suggest to add half the cylinder to the spherical component.

(c) 2022 The Author(s).

Published by S. Karger AG, Basel

\section{Introduction}

The prevalence of myopia is increasing $[1,2]$. In East Asia, 80-90\% of school-leavers are myopic [3]. Although Europeans are less affected, approximately $20 \%$ of Danish children aged 14-17 years are myopic [4]. Reduced visual acuity resulting from under-correction of myopia may enhance the progression of myopia $[5,6]$. Therefore, valid measurements of the refractive state in children are important for prescribing glasses and other vision correction modalities. Subjective manifest refraction (SR) determines the optimal correction for a refractive error [7]. This method depends on the feedback of the person tested while being presented with different lenses in a structured way. Accordingly, this method can be difficult to use on younger children, who often give inconsistent answers because of difficulties in keeping focus during the examination. karger@karger.com www.karger.com/bmh

Karger $\stackrel{\text { ' }}{5}$

BOPEN ACCESS
(C) 2022 The Author(s)

Published by S. Karger AG, Basel

This is an Open Access article licensed under the Creative Commons Attribution-NonCommercial-4.0 International License (CC BY-NC) (http://www.karger.com/Services/OpenAccessLicense), applicable to the online version of the article only. Usage and distribution for commercial purposes requires written permission.
Correspondence to:

Sara Bøgelund Rasmussen, sabr9@ hotmail.com 
The use of a computer-controlled autorefractor (AR) enables objective refraction. Most ARs use an internal fixation in conjunction with a built-in fogging mechanism to minimise accommodation, but readings may be unreliable due to proximal accommodation errors, especially in young children [8]. Accordingly, cycloplegic AR measurements are recommended for diagnosing refractive errors in children $<12$ years of age [9]. However, for the eye to obtain the best distance visual acuity, the state of accommodation should not be at infinity but at an intermediate distance in order to account for individual habitual accommodative tone. This accommodative tone or "lead" has been reported to vary between 0.15 dioptres (D) to approximately $1 \mathrm{D}[10,11]$. Accordingly, when making prescriptions using cycloplegic objective refraction the children are likely to experience less clear daily vision.

The Shin-Nippon Nvision-K 5001 AR, which is also known as Grand Seiko WR-5100K, is an open-field AR that can present the fixation object at any distance. In theory, placement of an object at optical infinity (i.e., $6 \mathrm{~m}$ ) should render measurements without accommodation and resemble the cycloplegic measurements of an ordinary AR, whereas placement of the fixation object at the most distant point without blur would resemble subjective refraction.

The purpose of this study was to explore if cycloplegic autorefraction is the best option as a starting point for prescribing the optimal correction for myopic children who cannot corporate for SR. In young myopic children with good cooperation, we determined the correlation between SR and (1) autorefraction with cycloplegia measured by the Shin-Nippon Nvision-K 5001, with the target object placed at the most distant point without blur, (2) autorefraction without cycloplegia measured by the Shin-Nippon Nvision-K 5001, with the target object placed at the most distant point without blur, and (3) autorefraction without cycloplegia measured by Topcon KR-800S with internal fixation.

\section{Materials and Methods}

\section{Study Design and Participants}

This cross-sectional comparative study was based on data collected from the randomised 18-month prospective 1:1 study entitled "Clinical study of near-sightedness, treatment with orthokeratology lenses (CONTROL-Study)" [12], which primarily aimed to investigate how nightly wear of orthokeratology lenses affected the progression of myopia compared to single-vision spectacles.

For this sub-study, 21 of the 60 subjects enrolled in the CONTROL-Study were eligible for analysis. The inclusion criteria were age 9-12 years with myopia of $0.5 \mathrm{D}$ to $4.75 \mathrm{D}$ spherical component and regular astigmatism up to $<2.5 \mathrm{D}$ in both eyes at the time of examination and completion of the relevant examinations either at baseline or on follow-up at 6 or 12 months. The exclusion criteria were use of orthokeratology lenses and a higher myopic spherical equivalent refractive error (SEQ) on cycloplegic autorefraction than on SR.

\section{Ophthalmologic Measurements}

All examinations were made at the University Hospital of Southern Denmark by the same ophthalmologist (TMJ). The relevant measurements for the present study included (1) monocular subjective manifest (non-cycloplegic) refraction using Jacksoncross cylinder technique [13] and applying maximum plus acceptance, (2) cycloplegic AR measurements, with the target object at the most distant point without blur, using the Shin-Nippon Nvision-K 5001, (3) non-cycloplegic AR measurements, with the target object at the most distant point without blur, using the ShinNippon Nvision-K 5001, and (4) non-cycloplegic AR measurements by Topcon KR-800S. The order of the examinations was protocolised and performed in the following order: SR, Shin-Nippon non-cycloplegic, Topcon non-cycloplegic, and Shin-Nippon cycloplegic. The Shin-Nippon Nvision-K 5001 and Topcon KR800 S examinations were performed on both eyes, the first always starting with the left eye and the latter always starting with the right eye.

For the Shin-Nippon Nvision-K 5001 measurements, a +5.00 D Badal lens was positioned on a tripod $20 \mathrm{~cm}$ in front of the eye (Fig. 1a). The target object (Fig. 1b) behind the Badal lens was moved on an optical bench (Fig. 1c) towards the subject until the most distant point without blur was reached (Fig. 1d). The fellow eye was covered. The target was illuminated by ambient room lighting supplemented by ceiling lights, resulting in a target luminance of $100 \mathrm{~cd} / \mathrm{m}^{2}$. A minimum of 5 readings were made for each measurement with the instrument aligned with the centre of the corneal reflex. Fixation was observed through the open-field screen, and measurements with unsteady fixation were deleted. Cycloplegia was achieved with the application of 3 drops of $1 \%$ cyclopentolate (Minims ${ }^{\circledR}$; Bausch \& Lomb, Chauvin Pharmaceuticals Ltd., UK) at 5-min intervals, with the last drop instilled 30-45 min prior to the cycloplegic AR measurements. The SEQ was calculated as the spherical power $+0.5 \times$ cylinder power.

\section{Statistical Analysis}

Data from right eyes were analysed and reported in order to avoid statistically testing on dependent data. Data with normal distribution are presented as means and standard deviations, and non-parametric data are presented as medians and ranges. To correct for multiple testing in the analysis of agreement between each variable in the measuring methods (SEQ, cylinder, and axes), the overall significance was tested using the one-way repeated analysis of variance (ANOVA) and ANOVA on ranks depending on the normal distribution of residuals for the variable in question. Only if the overall test was significant to the $5 \%$ level, the Tukey test was applied to test statistical significance between SEQ, cylinder, and axes of the SR against the same variables of the other 3 measurement methods. Agreement between subjective manifest SEQ and objective SEQs was graphically presented using Bland-Altman plots. Because correlations were suspected between the degree of myopia and the difference between the measurement methods, 


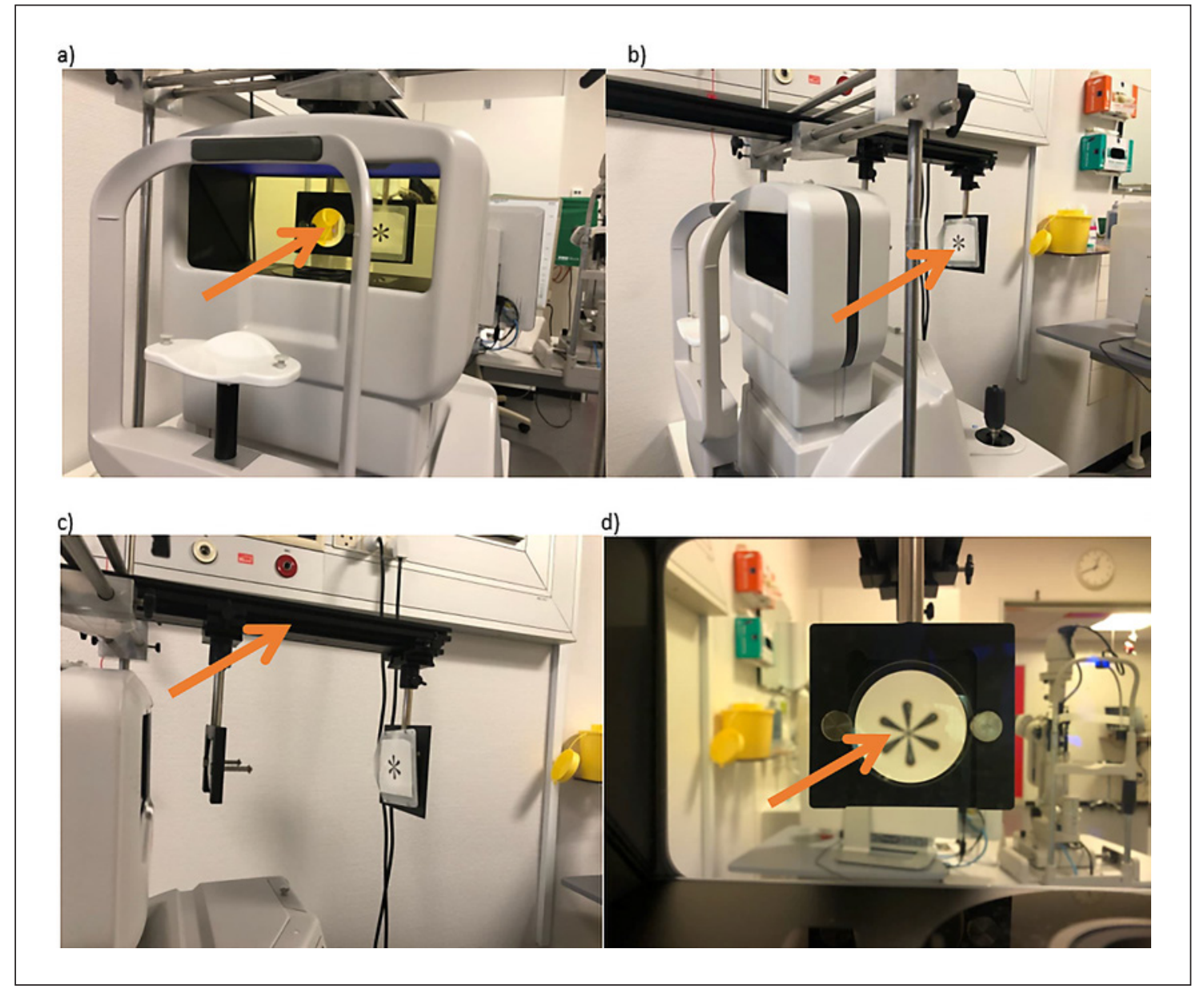

Fig. 1. The set-up of the AR Shin-Nippon Nvision-K 5001. The images show the Badal lens (orange arrow, a) as it points towards the fixation target (b) and optical bench (c) . d The fixation target is shown as viewed behind the Badal lens.

Table 1. Descriptive statistics and significance testing against SR for the spherical equivalent, cylinder, and axes

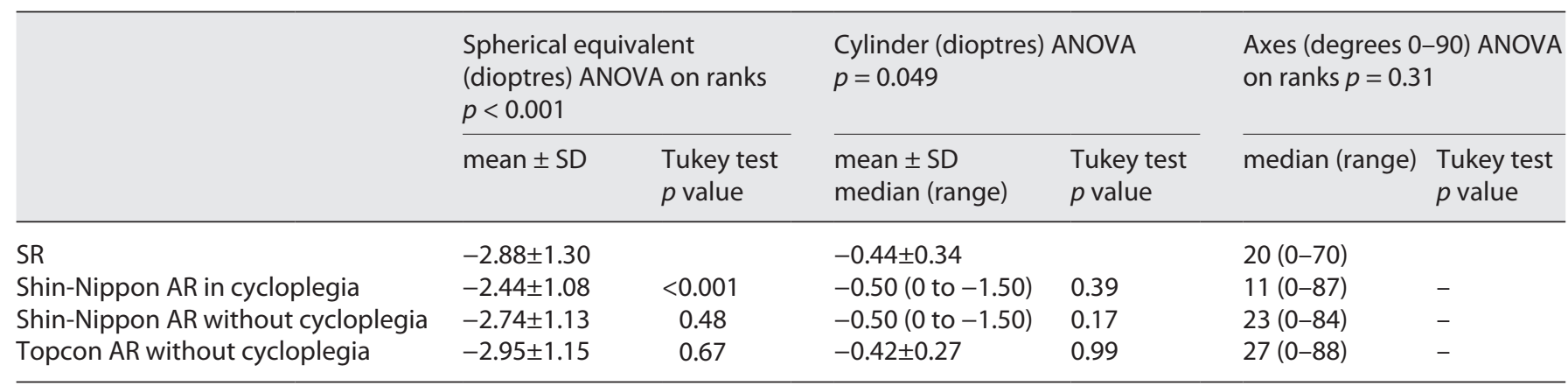

SD, standard deviation. When ANOVA/ANOVA on ranks was statistically significant to the $5 \%$ level, the Tukey test was performed to test for significance for each measure against SR. Since ANOVA on ranks for axes was not significant $(p=0.31)$, the Tukey test was not performed. 

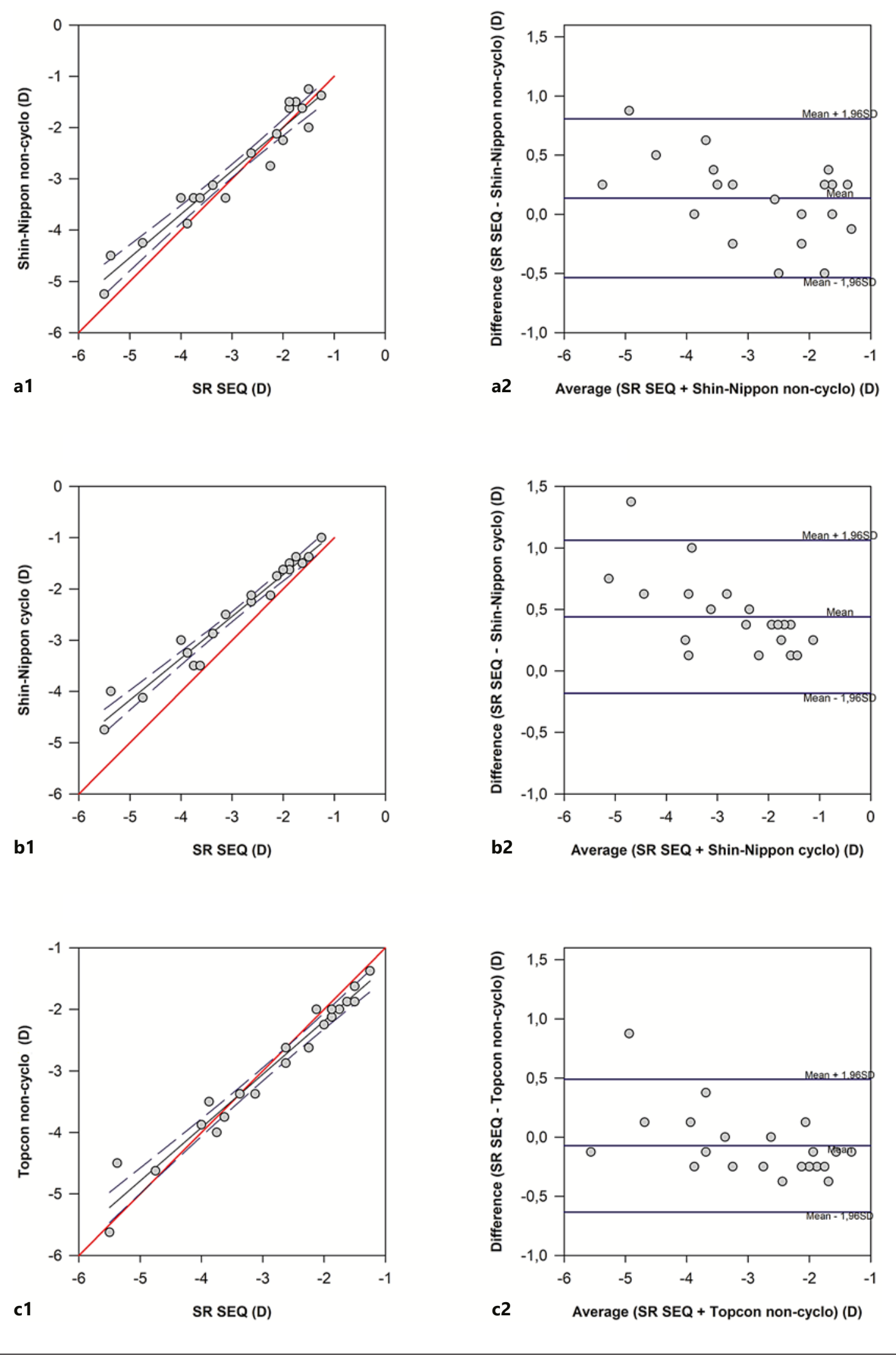

(For legend see next page.) 
linear correlations with Pearson's correlation coefficients were added to the plots. The $95 \%$ limits of agreement (LoAs) were defined as the mean \pm 1.96 standard deviation. A $p$ value of $\leq 0.05$ was considered significant. In order to detect an estimated clinically relevant difference of $0.38 \pm 0.5 \mathrm{D}$ between SR and cycloplegic AR measurements given a significance level of (alpha) of 0.05 and a statistical power $80 \%$, a sample size of 16 subjects in each group was required.

\section{Results}

The 21 subjects had a mean age of $10.62 \pm 0.81$ years; 13 were girls, and 8 were boys. The SEQ of the SR was significantly more myopic than the Shin-Nippon autorefraction measurements in cycloplegia $(-2.88 \pm 1.30$ vs. $-2.44 \pm 1.08, p<0.001$, ANOVA on ranks) (Table 1 ). There was no significant difference in SEQ between the SR and the non-cycloplegic AR measurements. For both measurements with and without cycloplegia, the Shin-

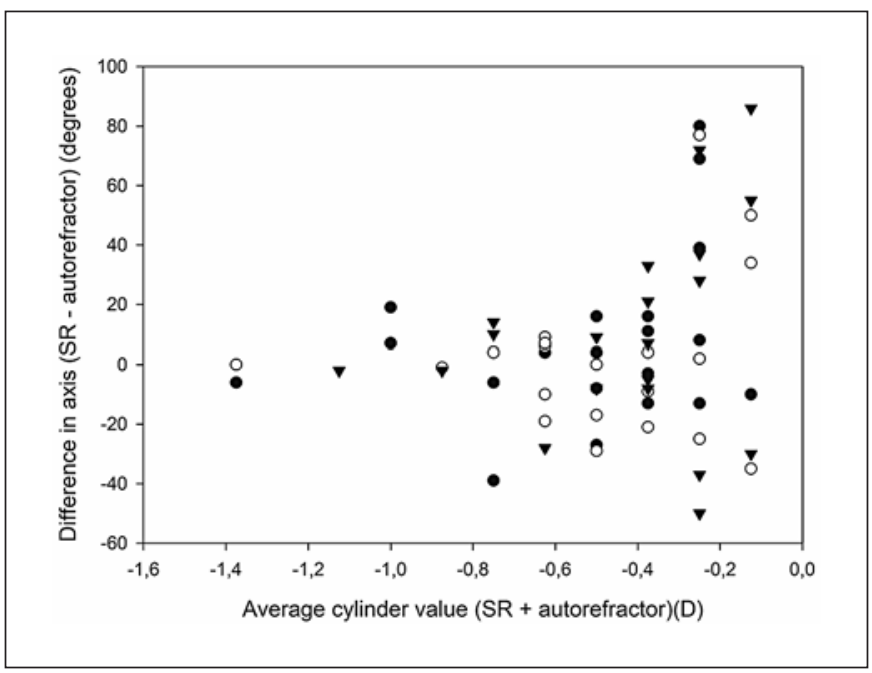

Fig. 3. The average cylinder values in dioptres of the SR and each of the AR values as a function of the axis difference $\left(0-90^{\circ}\right)$ between the SR and each of the AR measurements. Black circles, open circles, and black triangles represent average of SR and Shin-Nippon non-cycloplegic, Shin-Nippon cycloplegic, and Topcon noncycloplegic AR values, respectively. D, dioptres.

Fig. 2. Scatter plots and Bland-Altman plots between SEQ values of SR and Shin-Nippon non-cycloplegic AR values (a1-a2), SR and Shin-Nippon cycloplegic AR values (b1-b2), and SR and Topcon non-cycloplegic AR values (c1-c2). For the scatter plots, the red line indicates equality and the solid black line the regression line (a1: $r=-0.485, p<0.03$, Pearson's, b1: $r=-0.689, p<0.001$,

Achieving Optimal Correction for Young Myopic Children
Nippon measured a lesser degree of myopic refractive error (bias of $0.44 \mathrm{D}$ and $0.14 \mathrm{D}$, respectively; bias is the mean of the difference between the paired data) (shown in Fig. 2a2-b2). The bias of the Topcon AR was of $0.07 \mathrm{D}$ (shown in Fig. 2c2). The 95\% LoAs were $-0.18-1.06 \mathrm{D}$, $-0.53-0.87 \mathrm{D}$, and -0.63 to $0.49 \mathrm{D}$ for SR and, respectively, Shin-Nippon cycloplegic, Shin-Nippon non-cycloplegic, and Topcon AR values. A significant negative correlation $(p<0.05)$ was found between the data in each of the Bland-Altman plots (shown in Fig. 2a2-c2) indicating that all 3 methods showed a less myopic refractive error in moderate- to high-myopic subjects, whereas the 2 non-cycloplegic measurements tended to show a higher degree of myopia in low-myopic subjects. There was no significant difference in cylinder and axis values between SR and the 2 ARs (Table 1). However, the variation in axis measurements for both ARs was large for smaller cylinder values $(\leq 0.75 \mathrm{D})$ (shown in Fig. 3 ).

\section{Discussion}

In the present study, we found that cycloplegic autorefraction was significantly less myopic compared to SR. Non-cycloplegic measurements using the Topcon KR800 S resembled the SR measurements the most. This was surprising because we hypothesised that the measurements from an open-field AR, with the object placed in the most distant point without blur, would resemble SR, compared to an ordinary AR with an internal fixation object, which may be influenced by proximal accommodation errors. Furthermore, we found that the ARs both under cycloplegic and non-cycloplegic conditions tended to measure a lesser degree of myopic refractive error, especially in eyes with myopia of more than $-3.00 \mathrm{D}$. A study by Bamdad et al. [14] concurs our result that the cycloplegic autorefraction was significantly less myopic compared to SR.

Previous studies on the Shin-Nippon AR have shown varying results. One study showed a tendency for ShinNippon AR under non-cycloplegic conditions to overestimate myopia when compared with the estimation by SR

Pearson's, c1: $r=-0.530, p<0.01$, Pearson's), 95\% confidence intervals shown by dashed blue lines. For the Bland-Altman plots, the solid blue line indicates the bias, which is the mean of the difference between the paired data. The solid blue lines above and below show the $95 \%$ LoAs (mean $\pm 1.96 \mathrm{SD}$ ). SD, standard deviation; $\mathrm{D}$, dioptres. 
[8]. In the same study, cycloplegic autorefraction was comparable with the manifest subjective refraction. These findings were completely opposite to that of our results. In accordance with 2 other studies $[15,16]$, our study showed that the Shin-Nippon autorefraction resembled SR better without cycloplegia than with. However, the 95\% LoA for SR and Shin-Nippon non-cycloplegic was $-0.53 \mathrm{D}$ to $0.87 \mathrm{D}$ giving the risk of both over- and undercorrection when using these values for prescribing glasses.

Studies that validated both open-field $[17,18]$ and closed-field [14] ARs found slight overestimation of cylinder values. Our data showed no difference in cylinder values between $\mathrm{SR}$ and any of the AR measurements. However, all AR measurements showed bad correlations when determining the axis of cylinder values between $0.00 \mathrm{D}$ and $-0.75 \mathrm{D}$. Accordingly, we recommend the addition of cylindrical correction to the prescription, only when the value is more negative than $-0.75 \mathrm{D}$ because of the risks for overestimation of the value and misplacement of the axis. We recommend multiplying cylinders $<0.75 \mathrm{D}$ by 0.5 and adding this in the spherical component in order to maintain the SEQ.

Under-correction has been found to enhance the progression of myopia in myopic individuals $[5,6]$. In the study by Vasudevan et al. [5], the degree of under-correction was defined as the spherical equivalent between the subjective refraction for maximum visual acuity and the final prescription which ranged from $-0.13 \mathrm{D}$ to $-0.50 \mathrm{D}$. The study was retrospective and included 76 patients with a mean age of $14 \pm 5$ years. The follow-up period was 1 year. By correlation analysis, they found that the greater the degree of under-correction, the greater the myopic progression (measured by change in dioptres). The study by Chung et al. [6] was a randomized controlled trial on 94 children aged 9-14 years. In the intervention group, the children were under-corrected to a visual acuity of maximum distance monocular visual acuity of $6 / 12$ (20/40), while the control group was corrected to a distance monocular visual acuity of $6 / 6(20 / 20)$. After 2 -year follow-up, the progression in the under-corrected group was significantly higher as compared to the fully corrected group measured by both change in dioptre and axial length. In contrary, a 1-year prospective observational study on 253 Chinese grade 7 students found no difference in myopia progression between under-corrected and fully corrected children [19]. Under-correction was determined at baseline if visual acuity could improve 2 lines or more by subjective refraction. A resent systematic review on the topic advocate for full correction of myopia [20], and therefore, we find the results of our study important.

The application of the results of our study may provide better daily visual acuity for children who are unable to cooperate to SR; however, it may increase the risk for overcorrection in the aforementioned group of children. At present, the influence of overcorrection on the progression of myopia is unknown.

The main limitation for all the studies discussed, including this present study, was the use of SR as the gold standard for best visual acuity. The subjective nature of measurement may explain the variation in findings among the studies. The inclusion of patients aged 9-12 years only is also a limitation. Future studies with a wider age range and a larger number of children would be relevant. Another considerable limitation was that our study did not include cycloplegic measurements using Topcon KR-800S. Considering this study and previously published literature [8], an overcorrection may occur when non-cycloplegic autorefractions are used for prescriptions. Currently, it is unknown how overcorrection influences myopia development; however, studies have shown that under-correction enhances myopic progression [5, 6] and therefore may be considered more harmful. Longitudinal studies are needed to further investigate this.

In conclusion, when looking at the SEQ, non-cycloplegic autorefraction resembled SR the most. The measurements made with the Shin-Nippon Nvision-K 5001 AR in cycloplegia statistically significantly measured less myopic refractive errors, compared with those made by SR. Cylindrical values $<0.75 \mathrm{D}$ had invalid axis; accordingly, when prescribing glasses for children who cannot cooperate during SR, we suggest multiplying cylinders $<0.75 \mathrm{D}$ by 0.5 and adding this in the spherical component.

\section{Acknowledgment}

The authors acknowledge OPEN, Odense Patient Data Explorative Network, Odense University Hospital, Odense, Denmark, for data storage and data management support.

\section{Statement of Ethics}

All parents signed informed consents after receiving oral and written information from T.M.J. The study was carried out according to the Helsinki Declaration and Good Clinical Practice and has been reported to www.clinicaltrials.gov (NCT03246464). The study was approved by the Regional Committee of Health Research Ethics for Southern Denmark (Project-ID: S-20170002). 


\section{Conflict of Interest Statement}

The authors have no affiliations or financial conflicts of interest to declare.

\section{Funding Sources}

The CONTROL-Study was supported by grants from the Region of Southern Denmark; Forskningsrådet at University Hospital of Southern Denmark, Vejle Hospital; Bagenkop Nielsen Myopia Foundation; Fabrikant Einar Willumsen Foundation; Læge Frk. K. Rasmussen Foundation; Gangsted Foundation, Øjenforeningen; Overlæge Jørgen Werner Schous og hustru; Else-Marie Schou, født Wonge Foundation; Henry og Astrid Møller Foundation; and Grosserer Chr. Andersen og hustru Foundation.

\section{Author Contributions}

S.B.R., T.M.J., and F.M. contributed to the conception, design, and drafting of the study. T.M.J. collected data. S.B.R. analysed and interpreted data, with assistance from T.M.J. and F.M. T.M.J. and F.M. provided critical feedback to the work, and all authors helped shape the research, analyses, and manuscript. S.B.R., T.M.J., and F.M. approved the final version to be published and agree to be accountable for all aspects of the work.

\section{Data Availability Statement}

The data collected for this study are available through contacting the corresponding author.

\section{References}

1 Leo SW, Young TL. An evidence-based update on myopia and interventions to retard its progression. J AAPOS. 2011;15(2):181-9.

2 Dolgin E. The myopia boom. Nature. 2015; 519(7543):276-8

3 Lin LL, Shih YF, Hsiao CK, Chen CJ. Prevalence of myopia in Taiwanese schoolchildren: 1983 to 2000. Ann Acad Med Singap. 2004; $33(1): 27-33$.

4 Lundberg K, Suhr Thykjaer A, Sogaard Hansen R, Vestergaard AH, Jacobsen N, Goldschmidt E, et al. Physical activity and myopia in Danish children-The CHAMPS Eye Study. Acta Ophthalmol. 2018;96(2):134-41.

5 Vasudevan B, Esposito C, Peterson C, Coronado C, Ciuffreda KJ. Under-correction of human myopia - is it myopigenic? A retrospective analysis of clinical refraction data. J Optom. 2014;7(3):147-52.

6 Chung K, Mohidin N, O'Leary DJ. Undercorrection of myopia enhances rather than inhibits myopia progression. Vis Res. 2002;42(22): 2555-9.

7 Ferris FL 3rd, Kassoff A, Bresnick GH, Bailey I. New visual acuity charts for clinical research. Am J Ophthalmol. 1982;94(1):91-6.

8 Choong YF, Chen AH, Goh PP. A comparison of autorefraction and subjective refraction with and without cycloplegia in primary school children. Am J Ophthalmol. 2006; 142(1):68-74.
9 Fotedar R, Rochtchina E, Morgan I, Wang JJ, Mitchell P, Rose KA. Necessity of cycloplegia for assessing refractive error in 12-year-old children: a population-based study. Am J Ophthalmol. 2007;144(2):307-9.

10 Langaas T, Riddell PM, Svarverud E, Ystenaes AE, Langeggen I, Bruenech JR. Variability of the accommodation response in early onset myopia. Optom Vis Sci. 2008;85(1):37-48.

11 Plainis S, Ginis HS, Pallikaris A. The effect of ocular aberrations on steady-state errors of accommodative response. J Vis. 2005;5(5): 466-77.

12 Jakobsen TM, Moller F. Control of myopia using orthokeratology lenses in Scandinavian children aged 6 to 12 years. Eighteen-month data from the Danish Randomized Study: Clinical study Of Near-sightedness; TReatment with Orthokeratology Lenses $(\mathrm{CON}$ TROL study). Acta Ophthalmol. Epub 2021 Jul 7.

13 Jackson E. How to use the cross cylinder. Am J Ophthalmol. 1930;13(4):321-3.

14 Bamdad S, Momeni-Moghaddam H, Abdolahian M, Pinero DP. Agreement of wavefrontbased refraction, dry and cycloplegic autorefraction with subjective refraction. J Optom. 2020 Sep 4;S1888-4296(20)30098-4.
15 Davies LN, Mallen EA, Wolffsohn JS, Gilmartin B. Clinical evaluation of the Shin-Nippon NVision-K 5001/Grand Seiko WR-5100K autorefractor. Optom Vis Sci. 2003;80(4):320-4.

16 Cleary G, Spalton DJ, Patel PM, Lin PF, Marshall J. Diagnostic accuracy and variability of autorefraction by the Tracey Visual Function Analyzer and the Shin-Nippon NVision-K 5001 in relation to subjective refraction. Ophthalmic Physiol Opt. 2009;29(2):173-81.

17 Mallen EA, Wolffsohn JS, Gilmartin B, Tsujimura S. Clinical evaluation of the Shin-Nippon SRW-5000 autorefractor in adults. Ophthalmic Physiol Opt. 2001;21(2):101-7.

18 McCullough SJ, Little JA, Breslin KM, Saunders KJ. Comparison of refractive error measures by the IRX 3 aberrometer and autorefraction. Optom Vis Sci. 2014;91(10):118390.

19 Li SY, Li SM, Zhou YH, Liu LR, Li H, Kang MT, et al. Effect of undercorrection on myopia progression in 12-year-old children. Graefes Arch Clin Exp Ophthalmol. 2015; 253(8):1363-8.

20 Logan NS, Wolffsohn JS. Role of un-correction, under-correction and over-correction of myopia as a strategy for slowing myopic progression. Clin Exp Optom. 2020;103(2):1337. 